\title{
A New Approach to Detection of Systematic Errors in Secondary Substation Monitoring Equipment Based on Short Term Load Forecasting
}

\author{
Javier Moriano ${ }^{1}$, Francisco Javier Rodríguez ${ }^{1, *}$, Pedro Martín ${ }^{1}$, Jose Antonio Jiménez ${ }^{1}$ \\ and Branislav Vuksanovic ${ }^{2}$ \\ Received: 6 November 2015; Accepted: 7 January 2016; Published: 12 January 2016 \\ Academic Editor: Vittorio M. N. Passaro \\ 1 Department of Electronics, University of Alcalá, Alcalá de Henares, Madrid 28805, Spain; \\ javier.moriano@depeca.uah.es (J.M.); martin@depeca.uah.es (P.M.); jimenez@depeca.uah.es (J.A.J.) \\ 2 School of Engineering, University of Portsmouth, Winston Churchill Ave, Portsmouth PO1 3HJ, UK; \\ branislav.vuksanovic@port.ac.uk \\ * Correspondence: fjrs@depeca.uah.es; Tel.: +34-91-885-6561
}

\begin{abstract}
In recent years, Secondary Substations (SSs) are being provided with equipment that allows their full management. This is particularly useful not only for monitoring and planning purposes but also for detecting erroneous measurements, which could negatively affect the performance of the SS. On the other hand, load forecasting is extremely important since they help electricity companies to make crucial decisions regarding purchasing and generating electric power, load switching, and infrastructure development. In this regard, Short Term Load Forecasting (STLF) allows the electric power load to be predicted over an interval ranging from one hour to one week. However, important issues concerning error detection by employing STLF has not been specifically addressed until now. This paper proposes a novel STLF-based approach to the detection of gain and offset errors introduced by the measurement equipment. The implemented system has been tested against real power load data provided by electricity suppliers. Different gain and offset error levels are successfully detected.
\end{abstract}

Keywords: Short Term Load Forecasting (STLF); Artificial Neural Network (ANN); measurement error detection; secondary substation (SS)

\section{Introduction}

In the past few decades, significant advances in communication and information technology have accelerated the development and introduction of new broadband communication technologies in power systems. This fact has facilitated the power system automation in primary and secondary substations. With the aim of taking advantage of the current technology, there exists the IEC 61850 global standard for substation automation which is becoming particularly popular in recent years [1].

Nowadays, the development of Electronic Instrumental Transducer (EIT) and the importance of communication and information technology are quickly gaining acceptance in SSs. Consequently, traditional Substation Automation System (SAS) where Intelligent Electronic Devices (IEDs) were hardwire-linked are being challenged by this new protocol standardized as IEC 61850. Different EITs carry out the necessary current and voltage measurements, which are used to control and protect the SSs. However, the advantages that this approach brings could be negatively affected by the existence of erroneous measurements in the signal acquisition process [2].

On the other hand, the load profile and, therefore, the current consumption are strongly related to their historical measurement data depending on different variables, such as economic factors and environmental data. With the purpose of continuously maintaining the electricity generation and 
consumption balance, several approaches to load forecasting have been proposed during the last few years and some good examples can be found in [3,4].

In this paper, by using load forecasting techniques and comparing the forecast load with that from the measurement equipment in the SSs, different systematic error levels can be estimated, namely gain and offset errors. The implemented system is tested against data from both an SS simulator and electricity suppliers.

The scope of this work falls within two research fields: (1) short term load forecasting and (2) erroneous and false measurement detection. With this in mind, what follows presents the literature on both fields.

Different forecasting and prediction models have been employed in several fields, such as wind power prediction models [5] and photovoltaic power forecasting models [6]. As far as load forecasting is concerned, in literature, various approaches have been proposed to solve this issue. Load forecasting involves estimating the future electric load, for a forecast horizon, based on the available information about the state of the system. In this regard, this paper focuses on short term load forecast (STLF) to estimate the load with a horizon from one hour to one week. In [7], authors propose a new neural network approach to STLF based on a new modified learning algorithm. The results obtained are compared with those from other works $[8,9]$ with the aim of evaluating the validity of the proposed approach. In [10,11], environmental variables are also employed, whereas in $[12,13]$ only the historical load consumption is considered without a detrimental impact on the accuracy. The latter is the strategy adopted in this paper. In [12], the authors propose a solution for STLF in microgrids based on a three-stage architecture, which consists of a Self Organizing Map (SOM), a clustering via k-means algorithm, and a Multilayer Perceptron (MLP). A set of 29 inputs are provided to the MLP: 24 inputs that represent the hourly load of the previous day; two inputs for the day of the week of the previous day and two inputs for the month of the previous day in the form of sines and cosines; and lastly, one more input that represents the next day total load estimation. Finally, a total of 24 outputs corresponding to the estimated load for the forecasting day $(\mathrm{d})$ are obtained.

In [13], a different approach to one-day ahead load profile prediction is presented. The mean absolute percentage of the forecasting error is reduced between $0.5 \%$ and $16 \%$ by employing an Artificial Neural Network (ANN). Three different scenarios with different inputs are considered. The inputs to the ANN depend on the model implemented. For example, Model I requires 72 inputs, namely: hourly load values of the last day available, load values of the same weekday of previous week and load values of the same weekday two weeks before. The authors define a second model (Model II) by including three more inputs: two inputs represent the day of the week and the third one is used to distinguish between working day or vacation. In both models, 24 outputs are obtained which form the hourly predicted load for the forecast day. These outputs along with the 72 inputs of the first model could also be used as input of a new ANN with a total of 96 inputs (Model III).

Regarding erroneous measurement detection, this problem has been addressed in different papers. For example, in [14], the sensor failure detection is discussed employing a neural network. In [15] a method based on fractal dimension is implemented for sensor fault diagnosis. In [16], nonlinear principal component analysis (NLPCA) method for faulty measurement reconstruction is proposed. Finally, in [17], another neural-network-based algorithm is employed for sensor failure detection for a flight control system.

Focusing on SSs, in [2], pattern identification is utilized to detect erroneous measurements. In an attempt to achieve high pattern identification precision within the time limit imposed by the protection systems, a Radial Basis Function Neural Network (RBFNN) and an Orthogonal Least Square (OLS) learning algorithm are implemented. The neural network calculates the probability of fault occurrence based on measurements from all the EITs.

In order to provide a way of detecting a malfunction of an EIT in a particular SS, this work proposes a new approach to measurement error detection based on STLF. As mentioned before, in the literature, there are many examples of using STLF for monitoring and planning purposes and 
various approaches have been proposed to solve this issue. Interestingly, STLF has not been used for error detection until now. This strategy represents an innovative approach to error detection in SSs. This strategy greatly facilitates the detection of gain and offset error, thereby allowing preventive maintenance in the SSs to be performed. Particularly, the system presented in this paper could be effectively employed in open SS Nodes that are described in [18,19]. The open SS node is based on an architecture that combines smart metering with grid automation. While the EIT is operating, the developed system allows errors to be detected. In the event of occurring an error level higher than a threshold, an alarm can be raised and sent to the control center. Further tests for verifying the scope of the error could then be conducted by an operator.

This paper is divided into four sections. After the introduction, in Section 2, a global description of the system and the adopted methods are described. Section 3 analyzes both the data used to test the implemented system and the results achieved. Finally, some conclusions are drawn and future works are described in the final section.

\section{Methodology and System Description}

In this section, a general view on the employed system is discussed. Figure 1 shows an overview of the system. The available information pertains to the historical measurements from different SSs. The different electrical variables measured are: active and reactive power, voltage and current. However, for the system under study, just the current historical measurements are considered. This is due to the fact that the equipment for current measurement is more prone to errors on account of the considerable variability of the current.
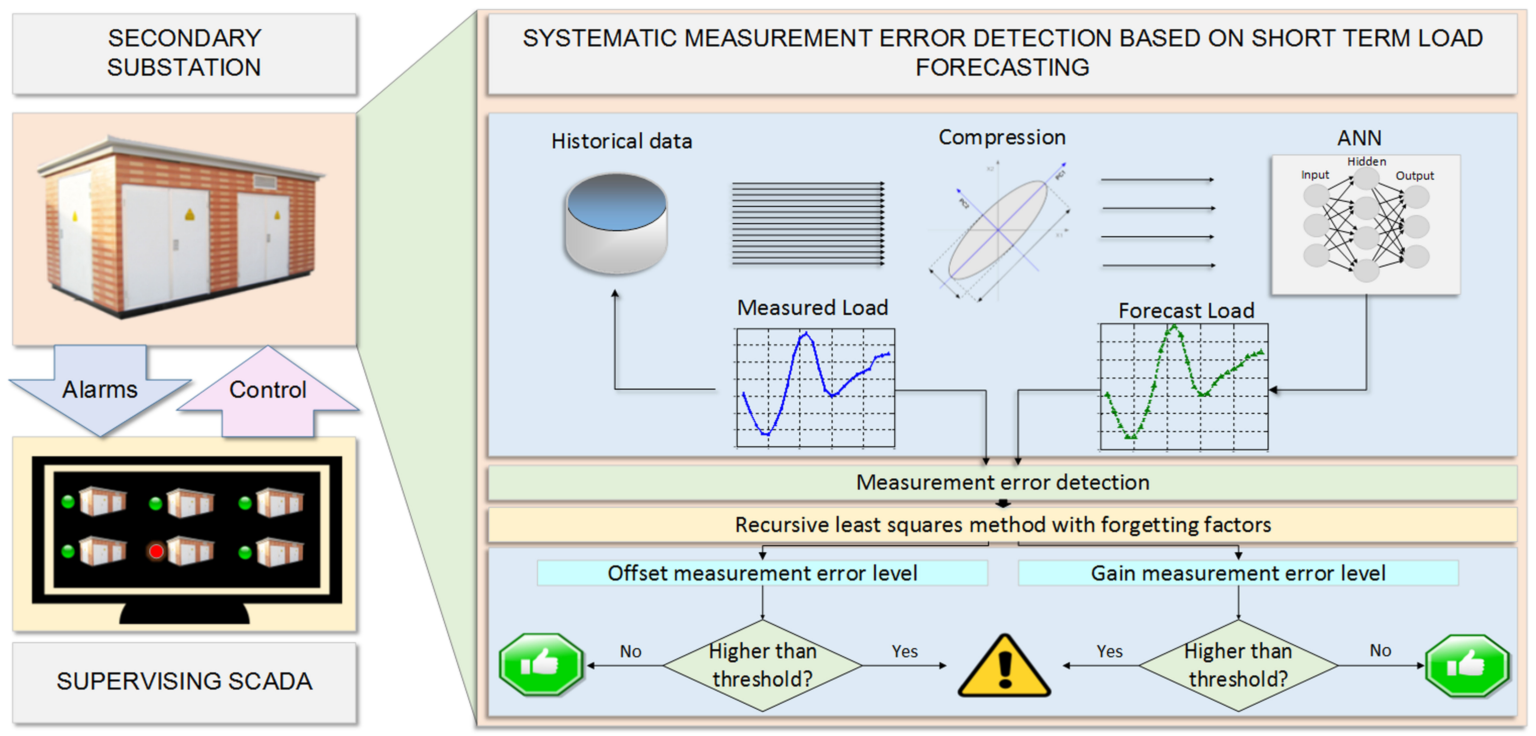

Figure 1. Overview of the general system.

The algorithm is divided into two steps: load forecasting and error estimation. Firstly, the available data is used to provide one-day ahead load forecast on a hourly basis. Secondly, once the measured load for the day under consideration is available, the error level is estimated by comparing both data sets, i.e., forecast and measured load.

\subsection{Load Forecasting}

As mentioned previously, the load forecasting model uses only historical measurement data, which consists of measurements from a set of SSs. Following one of the methods employed in [13], the system effectively performs, providing an accurate forecast. The chosen data, whereby the load profile on an hourly basis is estimated, is listed below: 
- $\quad 24 \mathrm{~h}$ load of the previous available day (d-1)

- $24 \mathrm{~h}$ load of the previous week same day $(\mathrm{w}-1)(\mathrm{d})$

- $24 \mathrm{~h}$ load of the two previous weeks same day $(\mathrm{w}-2)(\mathrm{d})$

- $\quad$ Day of the week (sine and cosine)

- Month of the year (sine and cosine)

Therefore, a total of 72 samples corresponding to hourly load are used. Since there exists a strong correlation among the 72 samples, a principal component analysis (PCA) procedure can be used to reduce the number of dimensions. This technique has been successfully employed in different fields such as face recognition [20], voice processing [21] and in ultrasonic sensors [22]. PCA basically is a statistical approach that linearly transforms an original set of variables into a smaller set of uncorrelated variables, which represent the largest variance from the multivariate input data.

The PCA algorithm has been described in [22,23]. Given a matrix $X$ with dimension $M \times N$, the first step consists in subtracting the mean of the elements in a particular row from each element of that row. Then, the covariance matrix from the previous matrix with the subtracted mean is obtained $(N \times N)$ and the eigenvectors and eigenvalues for that matrix are calculated. The eigenvectors with the highest eigenvalues represent the principal components $(k)$, and the number of principal components need to be determined. The set of eigenvectors corresponding to the principal components form the feature matrix $V$ and that can be expressed as:

$$
V=\left[\lambda_{1}, \lambda_{2}, \ldots, \lambda_{k}\right]_{(N \times k)}
$$

Finally, the new compressed matrix $(Y)$ is derived by multiplying the transposed feature matrix with the transposed zero-mean matrix previously calculated, as shown in the following equation:

$$
Y=\left[V^{T} \cdot \bar{X}^{T}\right]_{(k \times M)}
$$

The principal components resulting from the PCA process are the inputs of the ANN along with the data that represents the day of the week and the month of the year. Figure 2 shows the functional diagram of the system.

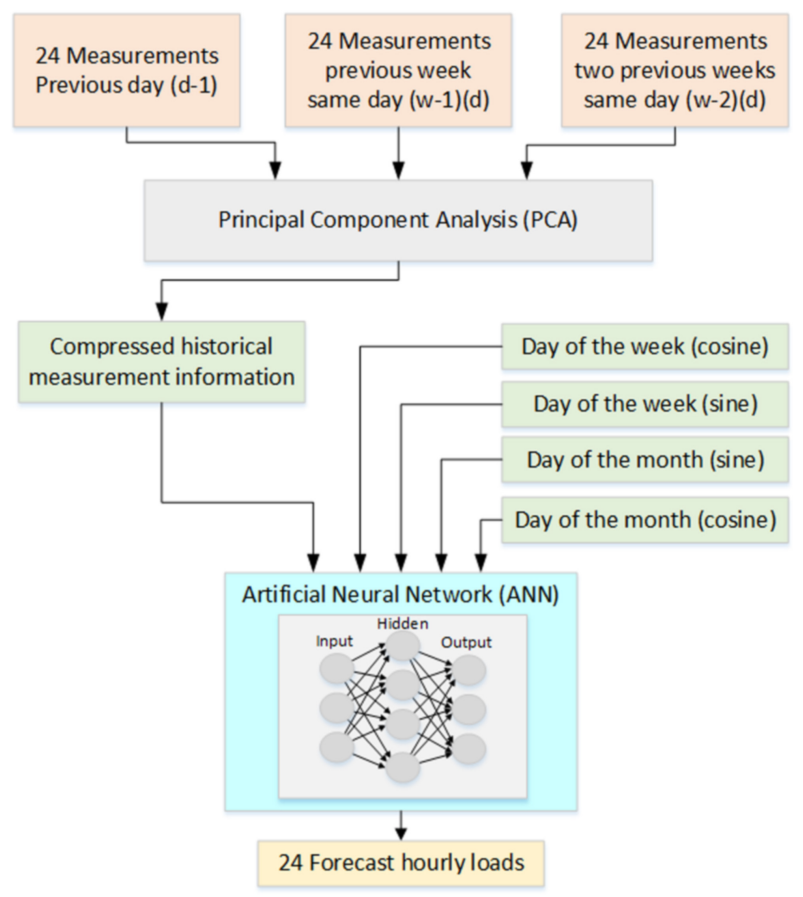

Figure 2. Functional diagram of the load forecasting system. 
As mentioned before, a set of 72-dimensional data provides historical information for the forecasting process of forecasting. Bearing in mind that the training process can be lengthy, the smaller the data set, the faster the process will be. Due to the strong correlation among the inputs, this data set can be described with only a few most significant components that hold $97 \%$ of the information, i.e., $97 \%$ of the variance for the principal components selected. In the system discussed in this paper, the number of principal components depends on whether the input data come from the simulator or from real measurements. This value ranges from seven to 20 components, being higher for the real data on account of less correlation.

The ANN used in this work is a one-hidden layer perceptron since it is the most frequently employed in forecasting and time series due to its performance [24]. The output and the input signals are related by the following equation:

$$
y=\varphi\left(\sum_{j=1}^{n} x_{j} \cdot w_{j}-\theta\right)
$$

where $y$ is the output, $x_{j}$ represents the input data, $w_{j}$ denotes the weight that is associated with each $x_{j}$, and $\theta$ is the threshold. Finally, $\varphi$ is the transfer function that is usually expressed as:

$$
\varphi=\frac{1}{1+e^{-x}}
$$

An important issue is to define the number of neurons of the hidden layer since this significantly affects the performance of the network in terms of execution time and accuracy. In this regard, an iterative process, which consists in carrying out different tests is used. Varying the number of neurons of the hidden layer from 1 to 100 and comparing the performance for each iteration, the ANN that minimizes the test error is composed of a 15-neuron hidden layer. Figure 3 shows the obtained mean square error (MSE) depending on the number of neurons in the hidden layer.

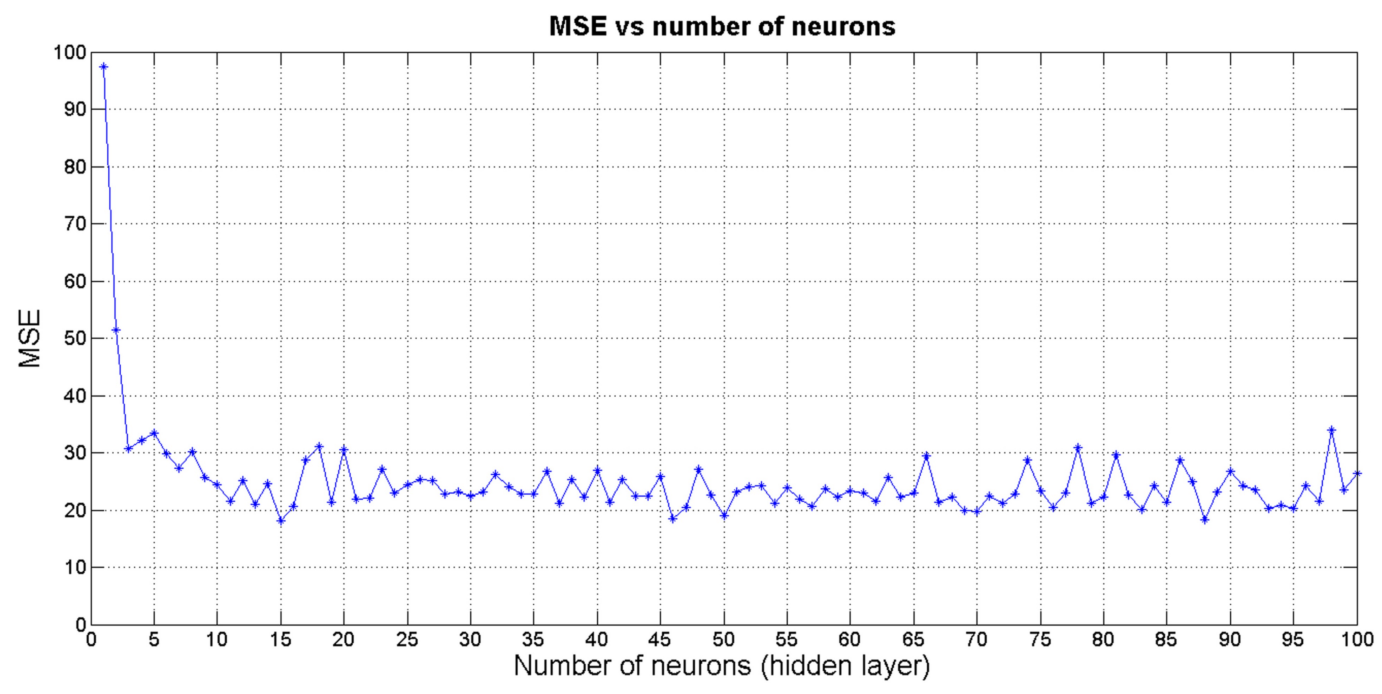

Figure 3. MSE depending on the number of neurons.

The Levenberg-Maquardt algorithm has been chosen for the ANN training for two reasons: (i) the number of iterations required for the ANN training is smaller in comparison to other techniques; (ii) this algorithm always guarantees the convergence of the training process $[25,26]$.

Taking into consideration that every EIT to be checked by the described system generates its own data, the ANN differs between the different EITs, hence the importance of selecting the algorithm that best provides accuracy and fast convergence in equal measure. 


\subsection{Error Measurement}

Once the load forecasting has been carried out and the new measurements for the day (d) are available, the process of error measurement detection for the different EITs can start. As stated in the introduction, the errors to be detected are systematic [27] and can be classified into two different types, namely: offset and gain errors.

Offset errors affect each acquired sample in a systematic way and hence they can question the accuracy of the measurements in the SS. The offset error in percentage is compared against the full scale. Figure 4 shows the load measurement deviation due to the offset.

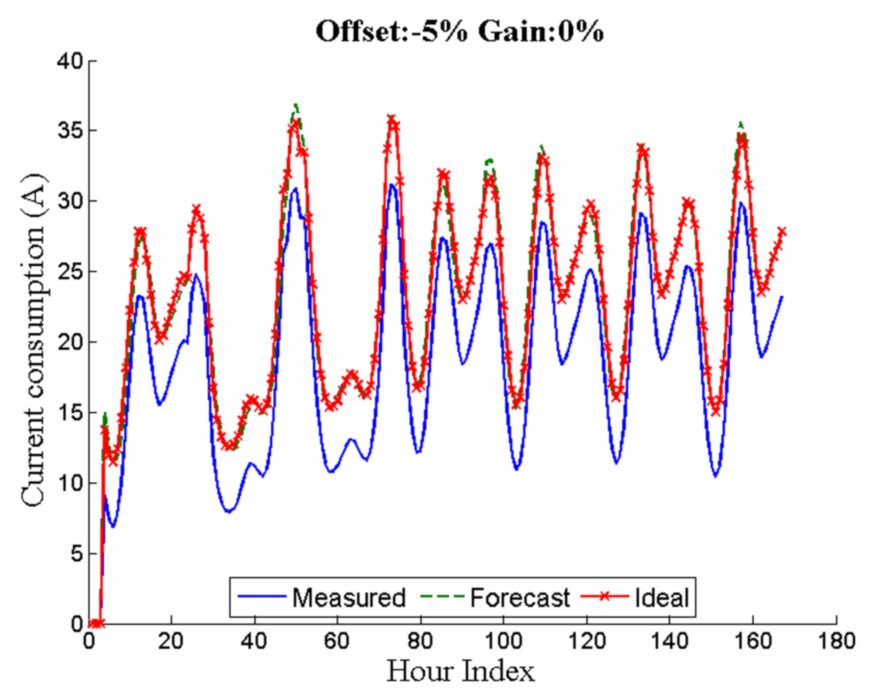

Figure 4. Seven days with an offset error of $-5 \%$.

Unlike offset errors, gain errors are multiplicative and affect all the measurements proportionally. Figure 5 shows a comparison between the measured, the forecast and the ideal load over a period of seven days for a gain error percentage of $-10 \%$.

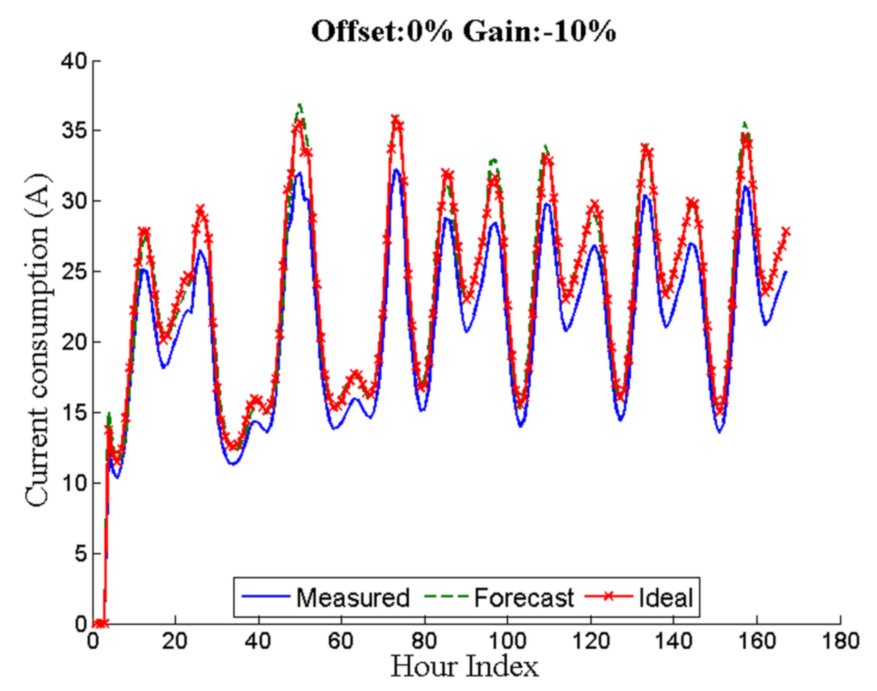

Figure 5. Seven days with a gain error of $-10 \%$.

Regarding their presence, offset and gain errors can occur simultaneously, which greatly hinders the error detection. In this context, both errors must be detected independently. Interestingly, 
the system described in this paper performs well under worst-case scenarios such as the occurrence of both errors with different signs. In this context, the most general possible scenario is considered when both errors appear at the same time as described in the following equation:

$$
m_{l p}(t)=\left(i_{l p}(t) \cdot \alpha\right)+\beta
$$

where $m_{l p}$ represents the measured load profile, $i_{l p}$ is the ideal measurement load profile, i.e., when no error occurs, and $\alpha$ and $\beta$ represent the gain and offset error, respectively.

By using the strategy described previously, the forecast load profile $\left(f_{l p}(t)\right)$ can be estimated. Since the process of forecasting introduces a random error $\left(e_{f}(t)\right)$, Equation $(5)$ can be rewritten as:

$$
\begin{aligned}
m_{l p}(t) & =\left(\left(f_{l p}(t)+e_{f}(t)\right) \cdot \alpha\right)+\beta \\
& =f_{l p}(t) \cdot \alpha+e_{f}(t) \cdot \alpha+\beta
\end{aligned}
$$

It goes without saying that the better the load prediction, the smaller the error introduced. However, this error can be negligible on account of the fact that it is a random error with mean 0 . Therefore, by using different averaging processes, Equation (7) can be reformulated as:

$$
\overline{m_{l p}}(t)=\overline{f_{l p}}(t) \cdot \alpha+\beta
$$

Differentiating both terms in Equation (8) leads to Equation (9):

$$
\frac{\Delta \overline{m_{l p}}(t)}{\Delta t}=\frac{\Delta\left(\overline{f_{l p}}(t) \cdot \alpha+\beta\right)}{\Delta t}=\frac{\Delta \overline{f_{l p}}(t)}{\Delta t} \cdot \alpha
$$

Then, the following equation can be obtained by solving for $\alpha$ :

$$
\alpha=\frac{\Delta \bar{m}_{l p}(t) / \Delta t}{\Delta \bar{f}_{l p}(t) / \Delta t}=\frac{\Delta \bar{m}_{l p}(t)}{\Delta \bar{f}_{l p}(t)}
$$

Finally, substituting the gain error into Equation (8), and solving for $\beta$ leads to Equation (11):

$$
\beta=\overline{m_{l p}}(t)-\overline{f_{l p}}(t) \cdot \alpha
$$

The proposed system is able to estimate the value of gain and offset error independently. Since there exists a considerable variability in the offset and gain measurement error estimation, the recursive least squares algorithm with forgetting factors (FRLS) is employed for filtering. This algorithm has been extensively utilized in the time-varying system as in [28] where a novel FRLS is developed. To be more precise, in this paper, a forgetting factor of 0.83 has been employed due to the performance enhancement on the trade-off between filtering and fast response.

\section{Simulations and Experimental Results}

With the aim of testing the system presented in this paper, two different data sets are used, namely: simulated data set from a simulator, and real data from SSs.

As far as the first data set is concerned, the simulator has been developed by a distribution operator, and it provides simulated measurements from different SSs located in the Henares Corridor, in Madrid. This simulator provides four measurements: active and reactive power, voltage and current. As mentioned in the introduction, only the current measurement is considered given that the equipment for current measurement is more prone to errors. Nevertheless, this system can be applied to the rest of the electrical variables.

A simulated data set based on current measurement is thus obtained every 15 min for two years (from 1 January 2010 to 31 December 2011). 
On the other hand, the system has also been tested against a real data set obtained from different SSs located in the Community of Madrid. This data set contains information half-hourly recorded from several SSs in the period ranging from 1 January 2010 to 31 December 2011.

Although both data sets contain data acquired with different frequencies, they are analyzed in the same way since only hourly average values are taken into consideration.

Then, the data sets are split into three groups: (i) the training subset (70\%), which is used to train the ANN; (ii) the validation subset (15\%) used to ensure that the network is generalizing and to stop training before overfitting; and (iii) the test subset (15\%) employed for a completely independent test of network generalization. Different error values of offset and gain are injected in the data set and then they are correctly detected. Likewise, the training time depends on whether the data is simulated or real. This stems from the fact that simulated data are more correlated and, therefore, fewer principal components are required.

The test was carried out in a computer with Intel Core i7 CPU $860 @ 2.93 \mathrm{GHz}$ x8 processor and the MATLAB neural network toolbox was employed.

\subsection{Results Based on the Simulated Data Set}

In Figures 6-8, the results obtained from testing the described system against simulated data are depicted. In Figure 6, all the measurements recorded over a two-year period (730 days-17,520 measurements) are shown.

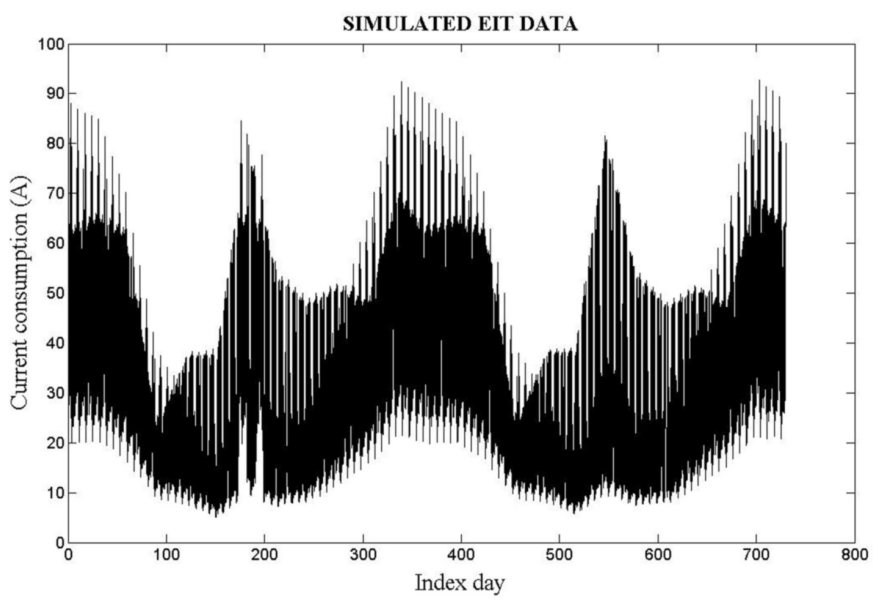

Figure 6. Simulated EIT-17,520 measurements obtained during 730 days.

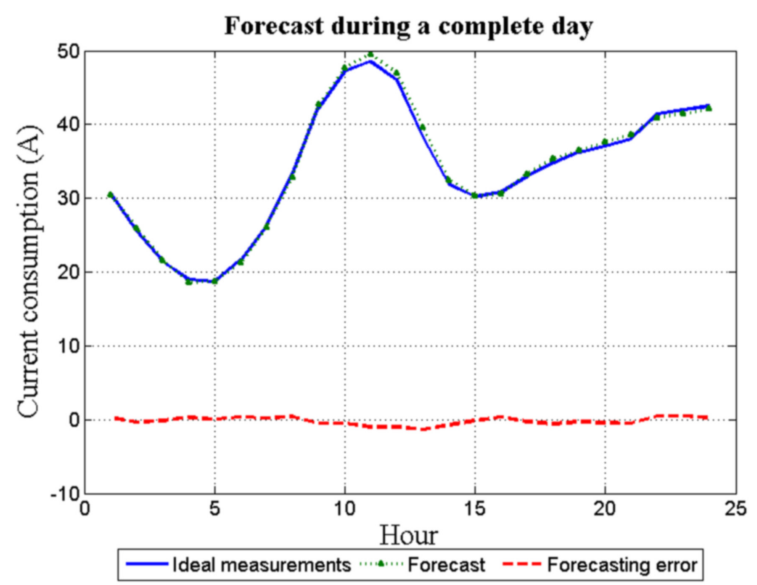

Figure 7. Simulated EIT-forecasting error for one-day ahead. 


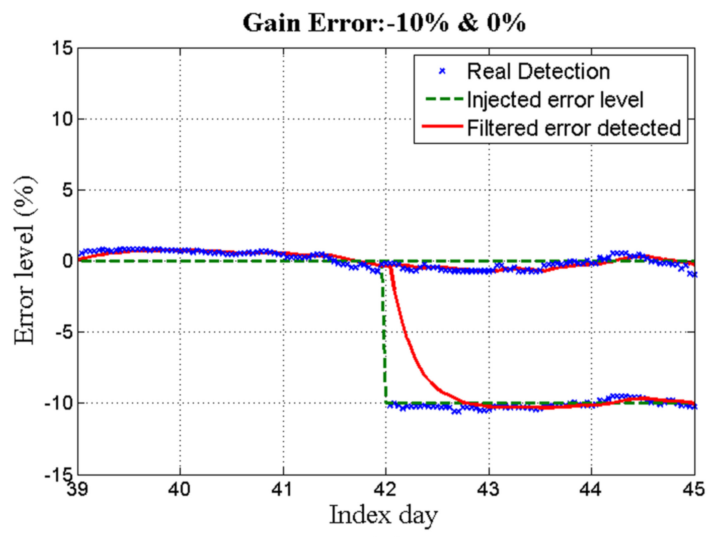

(a)

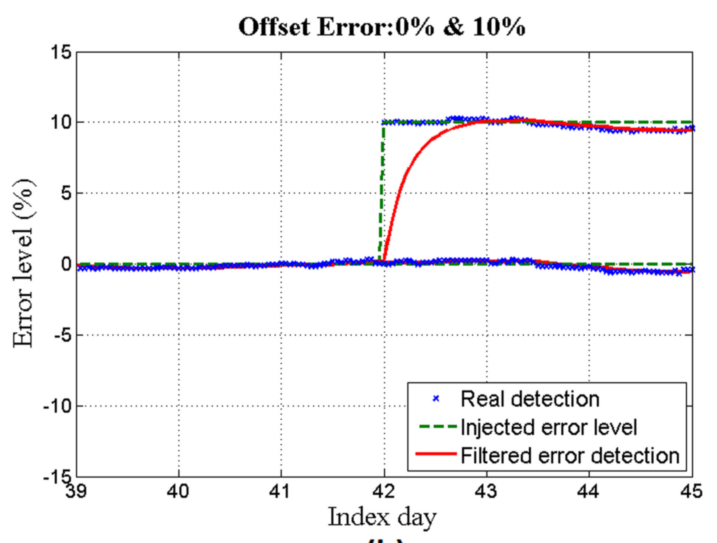

(b)

Figure 8. (a) $0 \%$ and $-10 \%$ gain errors and (b) $0 \%$ and $10 \%$ offset errors during the days after training.

Figure 7 shows both the 24 hourly forecast load for the day $d$ and the real measurements for the same day. Clearly, the error between them is negligible.

In Figure $8 \mathrm{a}, \mathrm{b}$ offset and gain measurement errors are injected respectively, and the system is applied to identify the level of error. It can be seen that different values of error for gain $(0 \%$ and $-10 \%)$ and offset $(0 \%$ and $10 \%)$ are successfully detected.

\subsection{Results Based on the Real Data Set}

In Figures 9 and 10a,b, the results obtained from testing the described system against real data are depicted.

Figure 9 shows for the same day $d$, both the 24 hourly forecast load and the available measurements. In this case, the error between them is not negligible. However, given that the forecasting error has a random nature and due to the different averaging processes, this error does not greatly affect the system discussed in this paper.

Finally, by injecting the same error levels as was done with the simulated data set, the system is applied to identify the error levels. Figure 10a,b represent the results achieved for offset and gain error measurements, respectively.

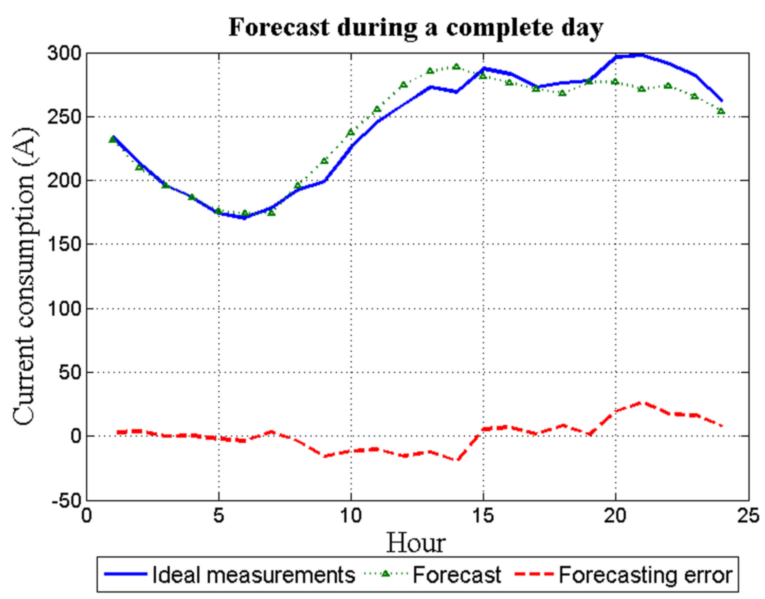

Figure 9. Real EIT—forecasting error for one-day ahead.

An error analysis strategy has been implemented [29]. The mean absolute percentage error (MAPE), the normalized mean bias error (NMBE) and normalized root mean square error (NRMSE) 
indicators are used to compare the results. These indicators show accuracy in terms of percentage and can be expressed as:

$$
\begin{gathered}
\text { MAPE }=\frac{1}{n} \cdot \sum_{i=1}^{n}\left|\frac{E_{i}-A_{i}}{E_{i}}\right| \times 100(\%) \\
N M B E=\frac{1}{n} \cdot \sum_{i=1}^{n}\left(\frac{E_{i}-A_{i}}{E_{i}}\right) \times 100(\%) \\
\text { NRMSE }=\sqrt{\frac{1}{n} \cdot \sum_{i=1}^{n}\left(\frac{E_{i}-A_{i}}{E_{i}}\right)^{2}} \times 100(\%)
\end{gathered}
$$

where $n$ denotes the number of samples, $E$ stands for the error level injected and $A$ represents the hourly detected error level.

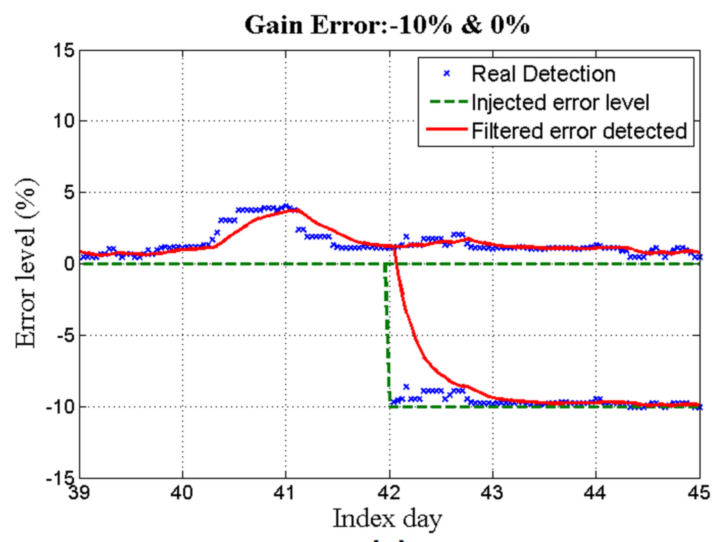

(a)

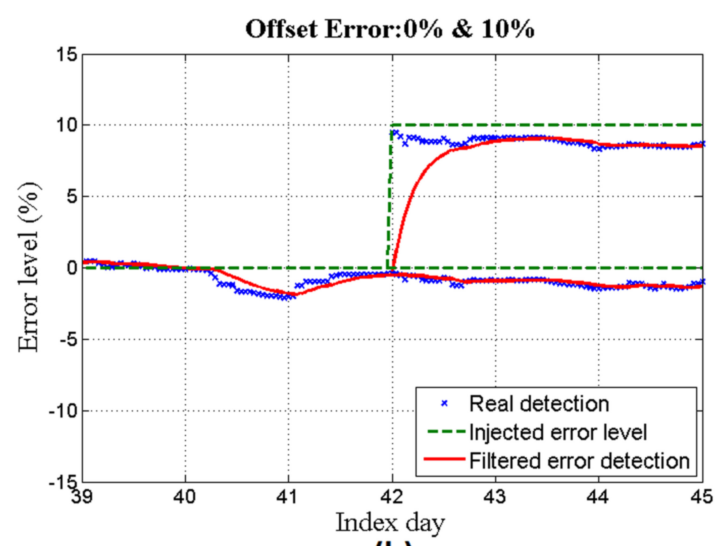

(b)

Figure 10. (a) $0 \%$ and $-10 \%$ gain errors and (b) $0 \%$ and $10 \%$ offset errors during the days after training.

Under normal operating conditions, the current measurement accuracy including all the equipment involved in the measuring process (mainly current transformer and measurement equipment) is below $\pm 2 \%$. The contribution to the error of the transformer is not higher than $\pm 1 \%$ [30,31] and the measurement equipment [32] can introduce between $\pm 0.5 \%$ and $\pm 1 \%$ of error depending on the manufacturer.

Within this context, for ensuring that a systematic measurement offset or gain error is occurring without generating false alarms, a minimum threshold of $\pm 5 \%$ is considered. Nonetheless, the results have been analyzed until a maximum measurement error of $\pm 10 \%$.

MAPE and NRMSE indicators show that there exists considerable variability in the result. However, the NMBE indicator is smaller, showing that the estimated error level oscillates around the right value. As a consequence, by using a recursive least squares algorithm with forgetting factor, the measured error estimation is filtered. Likewise, a comparison between the current and the filtered estimated errors in absolute value is drawn:

$$
a b s=|E-\bar{F}|
$$

where $a b s$ represents the absolute estimation error, $E$ stands for the injected error level and $\bar{F}$ is the mean value of the filtered estimated error level along the different tests. Tables 1 and 2 summarize the MAPE, NMBE, NRMSE and absolute errors indicators for gain and offset error in simulated data, respectively. Tables 3 and 4 show the same indicators for real data. 
Table 1. Error indicators for different gain error levels—Simulated data.

\begin{tabular}{ccccc}
\hline \multirow{2}{*}{ Error Level } & \multicolumn{4}{c}{ Simulated Data-Gain } \\
\cline { 2 - 5 } & MAPE (\%) & NMBE (\%) & NRMSE (\%) & Abs (\%) \\
\hline $\mathbf{- 1 0} \%$ & 20.915 & 17.021 & 25.857 & 1.705 \\
$\mathbf{- 9} \%$ & 21.629 & 16.357 & 27.001 & 1.472 \\
$\mathbf{- 8} \%$ & 22.871 & 15.718 & 28.786 & 1.264 \\
$\mathbf{- 7} \%$ & 24.970 & 15.353 & 31.574 & 1.076 \\
$\mathbf{- 6} \%$ & 27.958 & 14.930 & 35.393 & 0.895 \\
$\mathbf{- 5} \%$ & 32.548 & 14.694 & 41.157 & 0.741 \\
$\mathbf{+ 5} \%$ & 34.706 & 11.770 & 42.908 & 0.586 \\
$\mathbf{+ 6} \%$ & 29.723 & 11.694 & 36.719 & 0.697 \\
$\mathbf{+ 7} \%$ & 26.193 & 11.641 & 32.346 & 0.814 \\
$\mathbf{+ 8} \%$ & 23.615 & 11.558 & 29.150 & 0.920 \\
$\mathbf{+ 9} \%$ & 21.613 & 11.508 & 26.644 & 1.039 \\
$\mathbf{+ 1 0} \%$ & 20.068 & 11.513 & 24.702 & 1.152 \\
\hline
\end{tabular}

Table 2. Error indicators for different offset error levels-Simulated data.

\begin{tabular}{ccccc}
\hline \multirow{2}{*}{ Error Level } & \multicolumn{4}{c}{ Simulated Data-Offset } \\
\cline { 2 - 5 } & MAPE (\%) & NMBE (\%) & NRMSE (\%) & Abs (\%) \\
\hline $\mathbf{- 1 0} \%$ & 7.327 & 0.595 & 9.155 & 0.073 \\
$\mathbf{- 9} \%$ & 8.146 & 0.903 & 10.170 & 0.085 \\
$\mathbf{- 8} \%$ & 9.170 & 1.243 & 11.442 & 0.103 \\
$\mathbf{- 7} \%$ & 10.486 & 1.662 & 13.078 & 0.122 \\
$\mathbf{- 6} \%$ & 12.241 & 2.208 & 15.256 & 0.129 \\
$\mathbf{- 5} \%$ & 14.664 & 2.839 & 18.269 & 0.140 \\
$\mathbf{+ 5} \%$ & 14.870 & 3.845 & 18.732 & 0.195 \\
$\mathbf{+ 6} \%$ & 12.487 & 3.354 & 15.742 & 0.202 \\
$\mathbf{+ 7} \%$ & 10.761 & 2.955 & 13.573 & 0.207 \\
$\mathbf{+ 8} \%$ & 9.455 & 2.633 & 11.931 & 0.211 \\
$\mathbf{+ 9} \%$ & 8.426 & 2.362 & 10.636 & 0.212 \\
$\mathbf{+ 1 0} \%$ & 7.597 & 2.132 & 9.592 & 0.213 \\
\hline
\end{tabular}

Table 3. Error indicators for different gain error levels-Real data.

\begin{tabular}{ccccc}
\hline \multirow{2}{*}{ Error Level } & \multicolumn{4}{c}{ Real Data-Gain } \\
\cline { 2 - 5 } & MAPE (\%) & NMBE (\%) & NRMSE (\%) & Abs (\%) \\
\hline $\mathbf{- 1 0} \%$ & 29.476 & 11.584 & 35.924 & 1.156 \\
$\mathbf{- 9} \%$ & 32.658 & 11.662 & 39.666 & 1.047 \\
$\mathbf{- 8} \%$ & 36.603 & 11.560 & 44.344 & 0.925 \\
$\mathbf{- 7} \%$ & 42.024 & 11.582 & 50.772 & 0.809 \\
$\mathbf{- 6} \%$ & 48.871 & 11.561 & 58.913 & 0.690 \\
$\mathbf{- 5} \%$ & 58.561 & 11.506 & 70.410 & 0.574 \\
$\mathbf{+ 5} \%$ & 62.436 & 12.890 & 74.999 & 0.641 \\
$\mathbf{+ 6} \%$ & 52.737 & 13.088 & 63.483 & 0.784 \\
$\mathbf{+ 7} \%$ & 45.686 & 13.172 & 55.110 & 0.924 \\
$\mathbf{+ 8} \%$ & 40.366 & 13.091 & 48.779 & 1.045 \\
$\mathbf{+ 9} \%$ & 36.504 & 13.218 & 44.210 & 1.188 \\
$\mathbf{+ 1 0} \%$ & 33.438 & 13.407 & 40.601 & 1.344 \\
\hline
\end{tabular}

In order to carry out this test and in an attempt to enhance the versatility of this system, simulated and real data from five EITs located in different SSs is used. In this regard, 20 different tests are set for each EIT, which involves training a new ANN for each test.

Finally, with the aim of showing average results for each studied error under simulated and real scenarios, an averaging process over the tests and over the different EITs is performed. 
Table 4. Error indicators for different offset error levels-Real data.

\begin{tabular}{ccccc}
\hline \multirow{2}{*}{ Error Level } & \multicolumn{4}{c}{ Real Data-Offset } \\
\cline { 2 - 5 } & MAPE (\%) & NMBE (\%) & NRMSE (\%) & Abs (\%) \\
\hline $\mathbf{- 1 0} \%$ & 12.932 & 0.568 & 16.230 & 0.072 \\
$\mathbf{- 9} \%$ & 14.365 & 0.621 & 18.026 & 0.072 \\
$\mathbf{- 8} \%$ & 16.153 & 0.681 & 20.268 & 0.071 \\
$\mathbf{- 7} \%$ & 18.448 & 0.750 & 23.143 & 0.069 \\
$\mathbf{- 6} \%$ & 21.503 & 0.829 & 26.968 & 0.066 \\
$\mathbf{- 5} \%$ & 25.773 & 0.919 & 32.313 & 0.064 \\
$\mathbf{+ 5} \%$ & 25.576 & 3.271 & 31.930 & 0.146 \\
$\mathbf{+ 6} \%$ & 21.472 & 3.053 & 26.788 & 0.168 \\
$\mathbf{+ 7} \%$ & 18.517 & 2.822 & 23.090 & 0.183 \\
$\mathbf{+ 8} \%$ & 16.282 & 2.600 & 20.295 & 0.192 \\
$\mathbf{+ 9} \%$ & 14.527 & 2.394 & 18.103 & 0.198 \\
$\mathbf{+ 1 0} \%$ & 13.111 & 2.209 & 16.337 & 0.000 \\
\hline
\end{tabular}

Table 5 details the results obtained when both offset and gain errors occur simultaneously. In the authors' view, these results represent an excellent initial step toward the implementation of the system in open substation nodes for protection and security purposes.

Table 5. Combined error detection in real data

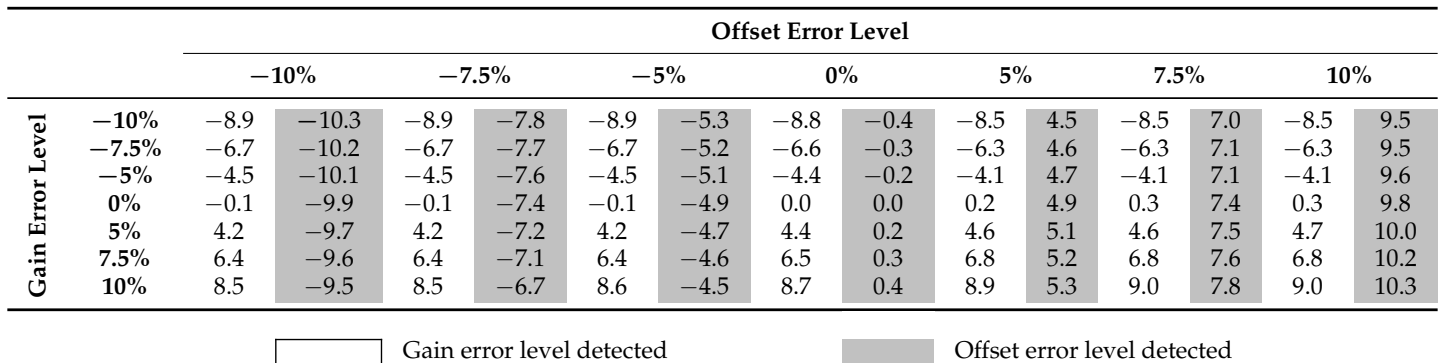

\section{Conclusions and Future Works}

Detection of measurement errors in electronic instrumental transducers (EITs) should play an important part in SS automation. Early detection of errors in the EITs can help not only reduce the risk of misoperation in the protection systems but also implement different preventive maintenance plans.

This paper has proposed a novel approach to measurement error detection based on short term load forecasting (STLF). Historical measurements acquired from EITs along with data related to the day and the month are the inputs to an ANN with the purpose of obtaining the forecast measurements for the day under consideration. Real and forecast measurements are then compared in order to independently detect offset and gain error levels.

The strength of the contribution made in this work lies in using STLF techniques to provide electricity companies with a valuable tool that serves as a decision-making aid in the area of maintenance and security. In this context, the system is designed to accurately detect offset and gain error levels introduced by EITs in SSs. This allows electricity companies to implement possible corrective and preventive actions.

Future work will concentrate on improving the forecasting model to deliver better results in terms of random errors detection and increased accuracy. With this in mind, different data, such as temperature, wind speed and economic rates could be included in the ANN training process.

Acknowledgments: This work has been supported by the Autonomous Community of Madrid under the PRICAM (Smart Grids Development Program for Autonomous Community of Madrid) project (S2013-ICE-2933), and the Spanish Ministry of Economy and Competitiveness under the CONPOSITE (Power Converters for the Optimization of the HVDC System Operation) project (ENE2014-57760-C2-2-R). 
Author Contributions: Francisco Javier Rodríguez and Pedro Martín conceived and designed the system. Javier Moriano and Jose Antonio Jiménez developed the algorithm and performed the simulations, and, finally, Branislav Vuksanovic analyzed the results. Javier Moriano and Francisco Javier Rodríguez drafted the manuscript. Pedro Martín, Jose Antonio Jiménez and Branislav Vuksanovic were involved in revision of the manuscript. All the authors checked the manuscript.

Conflicts of Interest: The authors declare no conflict of interest.

\section{References}

1. Schumacher, M.; Hoga, C.; Schmid, J. Get on the digital bus to substation automation. IEEE Power Energy Mag. 2007, 5, 51-56.

2. Sheng, S.; Xianzhong, D.; Chan, W.; Zhihuan, L. Erroneous measurement detection in substation automation system using OLS based RBF neural network. Int. J. Electr. Power Energy Syst. 2009, 31, 351-355.

3. Ho, K.L.; Hsu, Y.Y.; Yang, C.C. Short term load forecasting using a multilayer neural network with an adaptive learning algorithm. IEEE Trans. Power Syst. 1992, 7, 141-149.

4. Park, D.C.; El-Sharkawi, M.; Marks, R.; Atlas, L.; Damborg, M.J. Electric load forecasting using an artificial neural network. IEEE Trans. Power Syst. 1991, 6, 442-449.

5. De Giorgi, M.G.; Campilongo, S.; Ficarella, A.; Congedo, P.M. Comparison Between Wind Power Prediction Models Based on Wavelet Decomposition with Least-Squares Support Vector Machine (LS-SVM) and Artificial Neural Network (ANN). Energies 2014, 7, 5251-5272.

6. De Giorgi, M.G.; Congedo, P.M.; Malvoni, M. Photovoltaic power forecasting using statistical methods: Impact of weather data. IET Sci. Meas. Technol. 2014, 8, 90-97.

7. Amjady, N.; Keynia, F. A new neural network approach to short term load forecasting of electrical power systems. Energies 2011, 4, 488-503.

8. Huang, S.J.; Shih, K.R. Short-term load forecasting via ARMA model identification including non-Gaussian process considerations. IEEE Trans. Power Syst. 2003, 18, 673-679.

9. Amjady, N. Short-term hourly load forecasting using time-series modeling with peak load estimation capability. IEEE Trans. Power Syst. 2001, 16, 498-505.

10. Gulin, M.; Vasak, M.; Banjac, G.; Tomisa, T. Load forecast of a university building for application in microgrid power flow optimization. In Proceedings of the IEEE International Energy Conference, ENERGYCON 2014, Cavtat, Croatia, 13-16 May 2014; pp. 1223-1227.

11. Aung, Z.; Toukhy, M.; Williams, J.R.; Sanchez, A.; Herrero, S. Towards Accurate Electricity Load Forecasting in Smart Grids. In Proceedings of the Fourth International Conference on Advances in Databases, Knowledge and Data Application, Reunion Island, French, 29 February-5 March 2012; pp. 51-57.

12. Hernandez, L.; Baladrón, C.; Aguiar, J.M.; Carro, B.; Sanchez-Esguevillas, A.J.; Lloret, J. Short-Term Load Forecasting for Microgrids Based on Artificial Neural Networks. Energies 2013, 6, 1385-1408.

13. Sousa, J.; Neves, L.; Jorge, H. Assessing the relevance of load profiling information in electrical load forecasting based on neural network models. Int. J. Electr. Power Energy Syst. 2012, 40, 85-93.

14. Jäger, G.; Zug, S.; Brade, T.; Dietrich, A.; Steup, C.; Moewes, C.; Cretu, A.M. Assessing neural networks for sensor fault detection. In Proceedings of the 2014 IEEE International Conference on Computational Intelligence and Virtual Environments for Measurement Systems and Applications (CIVEMSA), Ottawa, ON, Canada, 5-7 May 2014; pp. 70-75.

15. Pengpeng, Q.; Jinguo, L.; Wei, Z.; Yangmin, L. Sensor fault diagnosis method based on fractal dimension. In Proceedings of the 2013 25th Chinese Control and Decision Conference (CCDC), Guiyang, China, 25-27 May 2013; pp. 2955-2960.

16. Harkat, M.F.; Djelel, S.; Doghmane, N.; Benouaret, M. Sensor fault detection, isolation and reconstruction using nonlinear principal component analysis. Int. J. Autom. Comput. 2007, 4, 149-155.

17. Napolitano, M.R.; Neppach, C.; Casdorph, V.; Naylor, S.; Innocenti, M.; Silvestri, G. Neural-network-based scheme for sensor failure detection, identification, and accommodation. J. Guid. Control Dyn. 1995, 18, 1280-1286.

18. Soriano, R.; Gonzalez, I.; Lugmaier, A.; Alberto, M.; Lorenzo, J.; Moreno, L. Opennode . Open Architecture for Secondary Nodes of the Electricity Smartgrid. Distribution 2011, 6, 6-9. 
19. Alberto, M.; Soriano, R.; Gotz, J.; Mosshammer, R.; Espejo, N.; Lemenager, F.; Bachiller, R. OpenNode: A smart secondary substation node and its integration in a distribution grid of the future. In Proceedings of the 2012 Federated Conference on Computer Science and Information Systems (FedCSIS), Wroclaw, Poland, 9-12 September 2012; pp. 1277-1284.

20. Swets, D.L. Using discriminant eigenfeatures for image retrieval. IEEE Trans. Pattern Anal. Mach. Intell. 1996, 18, 831-836.

21. Mami, Y.; Charlet, D. Speaker identification by anchor models with PCA/LDA post-processing. In Proceedings of the 2003 IEEE International Conference on Acoustics, Speech, and Signal Processing, (ICASSP'03), Hong Kong, China, 6-10 April 2003; Volume 1, pp. 1-180.

22. Jiménez, J.A.; Mazo, M.; Ureña, J.; Hernández, A.; Álvarez, F.; García, J.J.; Santiso, E. Using PCA in time-of-flight vectors for reflector recognition and 3-D localization. IEEE Trans. Robot. 2005, 21, 909-924.

23. Lim, S.T.; Yap, D.F.W.; Manap, N.A. Medical Image Compression Using Block-Based PCA Algorithm. In Proceedings of the 2014 International Conference on Computer, Communications, and Control Technology (I4CT), Langkawi, Malaysia, 2-4 September 2014; pp. 171-175.

24. Salcedo-Sanz, S.; Pérez-Bellido, A.M.; Ortiz-García, E.G.; Portilla-Figueras, A.; Prieto, L.; Correoso, F. Accurate short-term wind speed prediction by exploiting diversity in input data using banks of artificial neural networks. Neurocomputing 2009, 72, 1336-1341.

25. Chidrawar, S.K.; Bhaskarwar, S.; Patre, B.M. Implementation of neural network for generalized predictive control: A comparison between a newton Raphson and Levenberg Marquardt implementation. In Proceedings of the 2009 WRI World Congress on Computer Science and Information Engineering, CSIE 2009, Los Angeles, CA, USA, 31 March-2 April 2009; Volume 1, pp. 669-673.

26. Jia-Li, T.; Yi-Jun, L.; Fang-Sheng, W. Levenberg-marquardt neural network for gear fault diagnosis. In Proceedings of the 2010 2nd International Conference on Networking and Digital Society (ICNDS), Wenzhou, China, 30-31 May 2010; Volume 1, pp. 134-137.

27. Hofmann, D. Common Sources of Errors in Measurement Systems; Wiley Online Library: New York, NY, USA, 2005.

28. Yu, W.C.; Shih, N.Y. Bi-Loop Recursive Least Squares Algorithm. IEEE Signal Process. Lett. 2006, 13, 505-508.

29. De Giorgi, M.G.; Congedo, P.M.; Malvoni, M.; Laforgia, D. Error analysis of hybrid photovoltaic power forecasting models: A case study of mediterranean climate. Energy Convers. Manag. 2015, 100, 117-130.

30. Yamada, T.; Kon, S.; Hashimoto, N.; Yamaguchi, T.; Yazawa, K.; Kondo, R.; Kurosawa, K. ECT evaluation by an error measurement system according to IEC 60044-8 and 61850-9-2. IEEE Trans. Power Deliv. 2012, 27, 1377-1384.

31. Li, W.; Zhang, S.; Ma, W.; Ao, M. Application limitation of electronic current and Voltage Transformers in digital substations. In Proceedings of the 2011 IEEE Power Engineering and Automation Conference, PEAM 2011, Wuhan, China, 8-9 September 2011; Volume 2, pp. 496-499.

32. ZIV grid automation. 7IRD-E Distribution Protection \& Control Terminal. Available online: http://www. gridautomation.ziv.es/doc-downloads/literature/manuals/B7IR1107Ev00.pdf (accessed on 7 November 2015).

(C) 2016 by the authors; licensee MDPI, Basel, Switzerland. This article is an open access article distributed under the terms and conditions of the Creative Commons by Attribution (CC-BY) license (http://creativecommons.org/licenses/by/4.0/). 